\title{
Methylation status of RASSFIA associates with prognosis in metastatic gastric cancer
}

\begin{abstract}
Background: Cancer of the stomach represents one of the most common cancers all over the world. In these patients, several tumor suppressor genes are deactivated leading to tumor development. The deactivation of tumor suppressor genes, caused by hypermethylation of its promoters, has been correlated with poor prognosis in gastric cancer patients.
\end{abstract}

Materials and methods: In this study, the methylation status of the tumor suppressor gene RASSFI A was determined in the cell free DNA (cfDNA) of 55 patients with metastatic gastric cancer, using methylation specific polymerase chain reaction (PCR).

Results: The promoter region of RASSFIA gene was found to be methylated in 42 patients, but in none of the healthy control samples. Methylation of the promoter region of this gene was found to be correlated with shorter progression free survival (PFS, $p=0.026$ ) and overall survival $(\mathrm{OS}, \mathrm{p}=0.00 \mathrm{I})$ as compared to that of patients without promoter methylation.

Conclusions: These results are of interest and further studies are necessary to clarify whether hypermethylation of the RASSFIA tumor suppressor gene, could take a place as a biomarker for the early diagnosis, prognosis and prediction of response to treatment in patients with metastatic gastric cancer.
Volume 10 Issue 6 - 2019

\author{
Karamitrousis E,' Balgkouranidou I,' Xenidis \\ $\mathrm{N},{ }^{\prime}$ Amarantidis K,' Biziota E,' Koukaki T,' \\ Trypsianis G, ${ }^{2}$ Karayiannakis A, ${ }^{3}$ Bolanaki E. ${ }^{3}$ \\ Lianidou E, ${ }^{4}$ Kakolyris $\mathrm{S}^{\prime}$ \\ 'Department of Oncology, Democritus University of Thrace, \\ Greece \\ ${ }^{2}$ Department of Medical Statistics, Democritus University of \\ Thrace, Greece \\ ${ }^{3}$ Second Department of Surgery, Democritus University of \\ Thrace, Greece \\ ${ }^{4}$ Department of Chemistry, University of Athens, Greece
}

Correspondence: Evangelos Karamitrousis, Department of Oncology, Medical School, Democritus University of Thrace, Alexandroupolis, 68100, Greece, Tel +302551351092, Email vkaramitrousis@gmail.com

Received: October 22, 2019 | Published: December 12, 2019

Keywords: methylation, RASSFIA, gastric cancer, prognosis

Abbreviation: cfDNA, cell free DNA; OS, overall survival; $\mathrm{PCR}$, polymerase chain reaction; PFS, progression free survival; CTCs, circulating tumor cells; rpm, rounds per minute; sodium bisulfite

\section{Introduction}

Cancer of the stomach is a very common neoplasm. ${ }^{1}$ More than 27,000 new cases and 11,000 patients will die from this disease in the United States in 2019. ${ }^{2}$ In the Asian and African-American tribes, gastric cancer is a very common disease. The average age of diagnosis is about 70 years, with the majority of new cases occurring between the ages of 75-84years. The incidence has declined over the past decades. The outcome of advanced gastric cancer remains unfavorable, with a 5 -year survival rate of about $4 \% .^{3}$ The risk of developing gastric cancer decreases in people consuming fruits and vegetables, whereas the risk increases in people consuming large amounts of salt, and nitrosamines found in fried foods and processed meats. ${ }^{4}$ Helicobacter pylori, a bacterium located in the gastric mucosa, is associated with the development of gastritis, peptic ulcer and gastric cancer, whereas eradication of this bacterium appears to reduce the risk of gastric cancer. ${ }^{5,6}$ Epigenetics refers to inherited changes in gene expression, and one of the most well-known epigenetic events is methylation of DNA. In cancer, hypermethylation of tumor suppressor genes is a frequent event occurring in early or late stages of carcinogenesis. ${ }^{7}$ Indeed, a major event in carcinogenesis seems to be hypermethylation of $\mathrm{CpG}$ islands in the promoter region of tumor suppressor genes. ${ }^{8}$ This procedure affects the function of genes involved in many cell functions, such as the cell cycle and DNA repair, leading to the development of cancer. ${ }^{9}$ Moreover, each tumor type has specific hypermethylation patterns of $\mathrm{CpG}$ islands of the tumor suppressor genes. ${ }^{10}$ DNA methylation has a potential clinical significance as complementary diagnostic tool with prognostic and predictive role. ${ }^{11}$ In addition, the concentration of cell free DNA (cfDNA) in cancer patient's blood can be used as a biomarker to determine the origination of the tumor. ${ }^{12,13}$ cfDNA may be either free in the bloodstream or into circulating tumor cells (CTCs). ${ }^{15}$ Several studies have shown that cfDNA in the bloodstream is derived from the primary tumor cells. ${ }^{17}$ It is a valuable biologic material and has been utilized for the development of biomarkers with high sensitivity and specificity in cancer diagnosis. ${ }^{16}$ Regarding the predictive value of the cfDNA methylation pattern, several studies have been conducted in patients with bladder, colon and breast cancer. ${ }^{14}$ Epigenetic changes play an important role in the development and progression of gastric cancer and include DNA methylation, histone modifications, etc. Epigenetic alterations affect gene expression without modifying the DNA sequence and therefore are potentially reversible. ${ }^{18}$ RASSF1A is a major tumor suppressor gene that is frequently methylated in gastric cancer. ${ }^{19-21}$ The RASSF1A protein is actively involved in many cell functions, such as microtubule regulation, apoptosis modification, maintenance of genomic stability, cell cycle regulation, cellular mobility, and control of tumor invasiveness. ${ }^{22-24}$ In the present study, we examined the methylation status of RASSF1A gene and its possible associations with various clinicopathological parameters and overall survival of patients with metastatic gastric cancer.

\section{Materials and methods}

\section{Study design}

This is a single-institutional case-control study. The study material consisted of 55 blood samples obtained from gastric cancer patients suffering from metastatic disease. Inclusion criteria were: age over 
18years old, metastatic gastric adenocarcinoma. Exclusion criteria were: other gastric cancer histologies, except adenocarcinoma. Additionally, 20 blood samples taken from healthy individuals were used as a control group. All these control samples were taken from healthy friends and non-blood related family members of patients treated in the Department of Medical Oncology of the University Hospital of Alexandroupolis. The majority of them were age-matched with our patient population and received no medical care at the time of the sample collection.

\section{Sample collection and Isolation of Cell Free DNA}

Blood was collected in plasma collection tubes. Plasma was obtained immediately through centrifugation at 3,000 rounds per minute (rpm) for 10 minutes and stored at $-80^{\circ} \mathrm{C}$ until DNA extraction. Cell free DNA from serum samples was isolated using QIAamp DNA Blood mini kit (Qiagen, Germany) according to the manufacturer's protocol. DNA concentration was determined by a real-time PCR method using GAPDH gene as an amplifying target. Three microliters $(\mu \mathrm{l})$ of DNA elution were used as a template for the Sybr-green based real time PCR analysis. The cell free DNA concentration was calculated according to a reproducible standard dilution curves using a known concentration of MCF-7 genomic DNA, as previously described. ${ }^{20}$

\section{Sodium bisulfite conversion}

Extracted DNA was modified with sodium bisulfite (SB), in order to convert all unmethylated, but not methylated-cytosines to uracil. Bisulfite conversion was carried out up to $500 \mathrm{ng}$ of extracted DNA using the EZ DNA Methylation Gold Kit (ZYMO Research Co., Orange, CA), according to the manufacturer's instructions. The converted DNA was stored at $-80^{\circ} \mathrm{C}$ until used.

\section{Methylation specific PCR (MSP)}

The methylation status of RASSF1A gene in cell free circulating plasma DNA samples was detected by Real time MSP using specific primer pairs for both the methylated and unmethylated promoter sequences. The exact primer sequence for the methylated and unmethylated sequences is as follows:

\section{Unmethylated}

RASSF1A Forward 5'-GGT TGT ATT TGG TTG GAG TG 180

Reverse 3' - CTA CAA ACC TTT ACA CAC

AAC A

\section{Methylated \\ RASSF1A Forward 5' - GTT GGT ATT CGT TGG GCG C \\ Reverse 3' - GCA CCA CGT ATA CGT AAC G} 160

Amplification reactions were done in a Rotor Gene $\mathrm{Q}$, as previously described..$^{20,28}$

\section{Statistical analysis}

Statistical analysis of the data was performed using the Statistical Package for the Social Sciences (SPSS), version 21.0. The methylation status of RASSF1A gene, as well as all other qualitative variables was expressed as frequencies and percentages (\%). The chi-square test was used to evaluate any potential association of RASSF1A methylation status with patients' demographic and clinicopathological characteristics. Survival rates were calculated with the Kaplan-Meier method and the statistical difference between survival curves was determined with both log-rank and below tests. Multivariate Cox proportional hazards regression analysis was performed to explore the independent effect of RASSF1A methylation status on overall survival. Patients' gender, age, differentiation, and histologic subtype were also included in the multivariate model as potential confounders. All tests were two tailed and statistical significance was considered for $\mathrm{p}$ values $<0.05$.

\section{Results}

The methylation status of RASSF1A tumor suppressor gene was evaluated in the cfDNA in serum samples of 55 patients with metastatic gastric cancer and specifically in double-blind experiments. The clinicopathological characteristics of patients are reported in Table 1.

Table I Clinicopathological characteristics of patients and methylation status of RASSFIA gene

\begin{tabular}{llll}
\hline $\begin{array}{l}\text { Patient characteristics } \\
(\mathbf{n = 5 5 )}\end{array}$ & $\mathbf{n}(\%)$ & $\begin{array}{l}\text { RASSFIA } \\
\text { methylated }\end{array}$ & p value \\
\hline Gender & & & 0.256 \\
Females & $22(40.0 \%)$ & $17(77.3 \%)$ & \\
Males & $33(60.0 \%)$ & $25(75.6 \%)$ & \\
& & & 0.060 \\
Age & & & \\
& & & \\
$>60$ years & & & \\
& $17(30.9 \%)$ & $9(52.9 \%)$ & \\
Differentiation & $38(69.1 \%)$ & $33(86.8 \%)$ & \\
Moderate & & & \\
Poor & & & \\
Good & $22(40.0 \%)$ & $18(81.8 \%)$ & \\
Histology & $20(36.4 \%)$ & $16(80.0 \%)$ & \\
& $13(23.6 \%)$ & $8(61.5 \%)$ & \\
Intestinal & & & \\
Diffuse & & & \\
Other & $22(40.0 \%)$ & $17(77.3 \%)$ & \\
\hline
\end{tabular}

In the present study, the promoter of RASSF1A gene was found to be methylated in 42 out of 55 patients examined (76.3\%). In healthy control group, no promoter was found to be methylated $(0.0 \%)$. Patients who had methylated the promoter of RASSF1A gene, showed significantly lower PFS (17.9months) compared to patients in whom the gene promoter was unmethylated, and this was significant (40months, $\mathrm{p}=0.026$ ) (Figure 1). Patients who had methylated the RASSF1A gene showed significantly lower OS (24.2months) compared to patients in whom the gene promoter was unmethylated (48 months, $\mathrm{p}=0.001$ ) (Figure 2). Moreover, the methylation of the RASSF1A gene appeared to be more common in male and elderly patients ( $>60$ years old). It also appears to be associated with low and intermediate differentiation regarding tumor histology, as well as with a higher rate of intestinal type of gastric adenocarcinoma. However, the above results were not statistically significant $(\mathrm{p}=0.256, \mathrm{p}=0.060$, $\mathrm{p}=0.231$ and $\mathrm{p}=0.671$, respectively). 


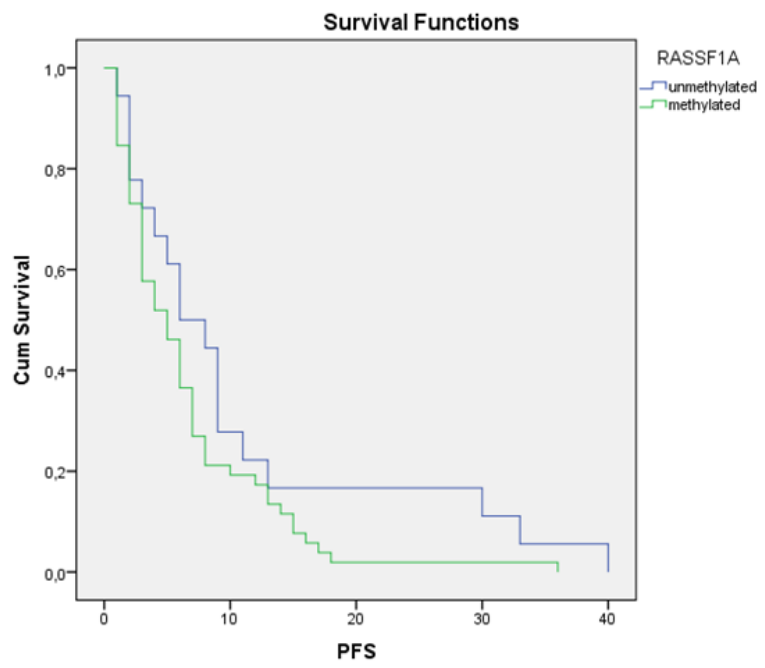

Figure I Correlation of RASSFIA gene methylation with progression free survival (PFS) $(p=0.026)$.

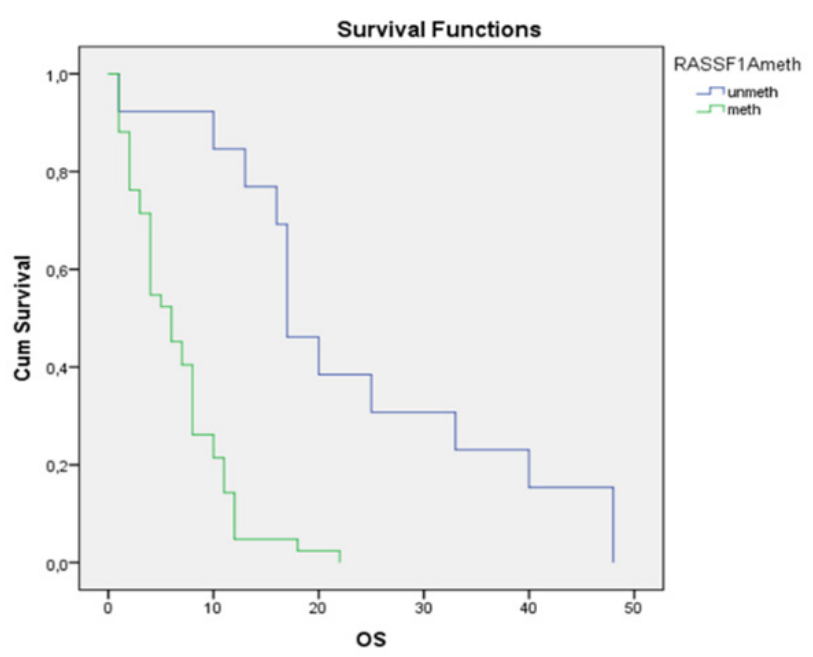

Figure 2 Correlation of RASSFIA gene methylation with overall survival (OS) $(p=0.00 I)$.

\section{Discussion}

Gastric cancer is one of the most common cancers in the world. In 2019 , it is estimated that there will be more than 27,000 new cases in the United States and more than 11,000 patients will die from the disease. At the time of diagnosis, the tumor is confined to the stomach, in $25 \%$ of the cases, in $20 \%$ is locally advanced and in $34 \%$ of the cases is already metastatic. Gastric cancer occurs more frequently in male patients, mainly in the Asian and AfricanAmerican tribes. The age of diagnosis is about 70years. The overall 5 -year survival of all patients with gastric cancer at any stage reaches $27.7 \%$. The incidence has declined over the past decades. However, despite advances in diagnosis and treatment, the outcome of advanced gastric cancer remains unfavorable, with a 5 -year survival rate of $4 \%$. Hypermethylation of $\mathrm{CpG}$ islands in the promoter region of tumor suppressor genes is a major event in carcinogenesis and it may affect the function of genes involved in the cell cycle, as well as other cell procedures. Hypermethylation occurs at different stages of cancer development, in different types of cells and is associated with genetic damage. The RASSF1A protein is involved in the regulation of cell cycle and microtubules, in the maintenance of genomic stability, in modification of apoptosis and cellular mobility. Finally, it seems that controls the tumor infiltration. The level of methylation of the corresponding gene in gastric cancer varies from $40-50 \%{ }^{24,25,29}$ In our study, the RASSF1A gene was methylated in 42 out of 55 patients $(76.3 \%)$, but in none of the 20 healthy individuals examined. This indicates that methylation of RASSF1A tumor suppressor gene is commonly seen in cancer patients but not in healthy individuals, suggesting that hypermethylation of the promoter of this gene could possibly serve as a potential biomarker for the early diagnosis of gastric cancer. There also appears to be a high degree of methylation of this gene in metastatic gastric cancer. This may be due to the crucial role of this gene not only in earlier but also in later stages of gastric cancer as in our study. This gene encodes a protein similar to the RAS family of effector proteins that act as tumor suppressors and its methylation is correlated with the development of gastric cancer. Inactivation of the gene, via methylation, is probably associated with a more aggressive tumor phenotype. This finding is in agreement with similar findings from other studies showing that hypermethylation of this gene is associated with poor survival..$^{25}$ In the present study, there was indeed an important correlation between gene hypermethylation and overall survival as well as progression free survival of patients with metastatic disease. The findings also come in line with those of other studies. ${ }^{26,27}$ In these studies, the incidence of hypermethylation of the RASSF1A gene promoter in the serum of gastric cancer patients was reported to be $25 \%$ and $34 \%$ respectively, which is considerably lower to the level observed in the present study (74.3\%). However, these variations between studies may be due to national, environmental and/or geographic factors that have established gastric cancer as a different disease in Europe and Asia. In addition, the number of samples analyzed ( $\mathrm{n}=55$ in the present study) is limited for safe statistical considerations. The sample number is relatively small. Therefore, no safe conclusions can be drawn, but there is a tendency towards the hypermethylation of the gene and the OS in these patients. This could be a weakness of the study. Moreover, the methylation of the RASSF1A gene appeared to be more common in male and elderly patients. In addition RASSF1A hypermethylation was more common in patients with low and intermediate differentiation regarding tumor histology, and with a higher rate of intestinal type of gastric adenocarcinoma. However, these findings never reached statistical significance. These results show that methylation of RASSF1A may be mostly a late event in gastric cancer patients and as such, may be associated with a more aggressive tumor phenotype. In conclusion, this study showed that hypermethylation of the promoter region of tumor suppressor gene RASSF1A, is a frequent epigenetic phenomenon in patients with advanced gastric cancer. A significant correlation between methylation of the promoter region of these genes and the PFS as well as the OS, was also observed. MSP is an inexpensive and feasible and reproducible method that can be applied in most clinical laboratories and can be useful in daily clinical practice for the diagnosis and prognosis of gastric cancer patients. This is strength of the present study. More studies with larger number of patients are needed to establish tumor suppressor gene RASSF1A as a potential biomarker for early diagnosis, prognosis and prediction of response to chemotherapy in gastric cancer patients.

\section{Conclusions}

In this study, the correlation of methylation of the tumor suppressor RASSF1A gene with the prognosis of patients with metastatic gastric cancer was demonstrated. The methylation of the gene appears to be 
associated with decreased OS and PFS of these patients. In the future, it may be a promising biomarker for this disease.

\section{Acknowledgments}

None.

\section{Conflicts of interest}

Author declares that there are no conflicts of interest.

\section{References}

1. Jemal A, Bray F, Center MM, et al. Global cancer statistics. CA Cancer J Clin. 2011;61(2):69-90.

2. Siegel RL, Miller KD, Jemal A. Cancer statistics, 2019. CA Cancer J Clin. 201;69(1):7-34.

3. SEER Cancer Statistics Review (CSR) 1975-2014. SEER. 2018.

4. Bertuccio P, Rosato V, Andreano A, et al. Dietary patterns and gastric cancer risk: a systematic review and meta-analysis. Ann Oncol. 2013;24(6):1450-1458.

5. Ma JL, Zhang L, Brown LM, et al. Fifteen-year effects of Helicobacter pylori, garlic, and vitamin treatments on gastric cancer incidence and mortality. J Natl Cancer Inst. 2012;104(6):488-492.

6. Fuccio L, Zagari RM, Eusebi LH, et al. Meta-analysis: can Helicobacter pylori eradication treatment reduce the risk for gastric cancer? Ann Intern Med. 2009;151(2):121-128.

7. Esteller M, Almouzni G. How epigenetics integrates nuclear functions. Workshop on epigenetics and chromatin: transcriptional regulation and beyond. EMBO Rep. 2005;6(7):624-628.

8. Greger V, Passarge E, Höpping W, et al. Epigenetic changes may contribute to the formation and spontaneous regression of retinoblastoma. Hum Genet. 1989;83(2):155-158.

9. Herman JG, Baylin SB. Gene silencing in cancer in association with promoter hypermethylation. N Engl J Med. 2003;349(21):2042-2054.

10. Esteller M. Epigenetics in cancer. N Engl J Med. 2008;358(11):11481159 .

11. Lee WH, Morton RA, Epstein JI, et al. Cytidine methylation of regulatory sequences near the pi-class glutathione S-transferase gene accompanies human prostatic carcinogenesis. Proc Natl Acad Sci USA. 91(24):11733-11737.

12. Hoque MO, Feng Q, Toure P, et al. Detection of Aberrant Methylation of Four Genes in Plasma DNA for the Detection of Breast Cancer. J Clin Oncol. 2006;24(26):4262-4269.

13. Wong TS, Man MWL, Lam AKY, et al. The study of p16 and p15 gene methylation in head and neck squamous cell carcinoma and their quantitative evaluation in plasma by real-time PCR. Eur J Cancer. 2003;39(13):1881-1187.

14. Levenson VV. DNA methylation as a universal biomarker. Expert Rev Mol Diagn. 2010;10(4):481-488.
15. Alix-Panabières $\mathrm{C}$, Schwarzenbach $\mathrm{H}$, Pantel K. Circulating tumor cells and circulating tumor DNA. Annu Rev Med. 2012;63:199-215.

16. Kohler C, Barekati Z, Radpour R, Zhong XY. Cell-free DNA in the circulation as a potential cancer biomarker. Anticancer Res. 2011;31(8):2623-2628.

17. Jin H, Ma Y, Shen Q, et al. Circulating Methylated DNA as Biomarkers for Cancer Detection. Methylation - DNA RNA Histones Dis Treat. 2012 .

18. Chen HM, Fang JY. Epigenetic Biomarkers for the Early Detection of Gastrointestinal Cancer. Gastrointest Tumors. 2014;1(4):201-208.

19. Liu JB, Wu XM, Cai J, et al. CpG island methylator phenotype and Helicobacter pylori infection associated with gastric cancer. World J Gastroenterol WJG. 2012;18(36):5129-5134.

20. Balgkouranidou I, Matthaios D, Karayiannakis A, et al. Prognostic role of APC and RASSF1A promoter methylation status in cell free circulating DNA of operable gastric cancer patients. Mutat Res. 2015;778:46-51.

21. Balgkouranidou I, Karayiannakis A, Matthaios D, Bolanaki H, Tripsianis G, Tentes AA, et al. Assessment of SOX17 DNA methylation in cell free DNA from patients with operable gastric cancer. Association with prognostic variables and survival. Clin Chem Lab Med. 2003;51(7):1505-1510.

22. Ghazaleh HA, Chow RS, Choo SL, Pham D, Olesen JD, Wong RX, et al. 14-3-3 mediated regulation of the tumor suppressor protein, RASSF1A. Apoptosis. 2010;15(2):117-127.

23. Shivakumar L, Minna J, Sakamaki T, Pestell R, White MA. The RASSF1A tumor suppressor blocks cell cycle progression and inhibits cyclin D1 accumulation. Mol Cell Biol. 2002;22(12):4309-4318.

24. Dallol A, Agathanggelou A, Fenton SL, et al. RASSF1A interacts with microtubule-associated proteins and modulates microtubule dynamics. Cancer Res. 2004;64(12):4112-4116.

25. Yao D, Shi J, Shi B, et al. Quantitative assessment of gene methylation and their impact on clinical outcome in gastric cancer. Clin Chim Acta. 2012;413(7-8):787-794

26. Wang $\mathrm{YC}, \mathrm{Yu} \mathrm{ZH}$, Liu $\mathrm{C}$, et al. Detection of RASSF1A promoter hypermethylation in serum from gastric and colorectal adenocarcinoma patients. World J Gastroenterol. 2008;14(19):3074-3080.

27. Tan SH, Ida H, Lau QC, et al. Detection of promoter hypermethylation in serum samples of cancer patients by methylation-specific polymerase chain reaction for tumour suppressor genes including RUNX3. Oncol Rep. 2007;18(5):1225-1230.

28. Fackler MJ, McVeigh M, Evron E, et al. DNA methylation of RASSF1A, HIN-1, RAR-beta, Cyclin D2 and Twist in in situ and invasive lobular breast carcinoma. Int J Cancer. 2003;107(6):970-975.

29. Pfeifer GP, Dammann R. Methylation of the tumor suppressor gene RASSF1A in human tumors. Biochemistry (Mosc). 2005;70(5):576583. 